\title{
Complex Billiard Hamiltonian Systems and Nonlinear Waves
}

\author{
Mark S. Alber * Gregory G. Luther ${ }^{\dagger} \quad$ Jerrold E. Marsden ${ }^{\ddagger}$
}

November, 1995; this version January 5, 1996

\section{In memory of Irene Dorfman}

\begin{abstract}
The relationships between phase shifts, monodromy effects and billiard solutions are studied in the context of Riemann surfaces for both integrable ordinary and partial differential equations. The ideas are illustrated with the three wave interaction, the nonlinear Schrödinger equation, a coupled Dym system and the coupled nonlinear Schrödinger equations.
\end{abstract}

\section{Introduction}

There is a deep connection between solutions of nonlinear equations and both geodesic flows and billiards on Riemannian manifolds. For example, in Alber, Camassa, Holm and Marsden [1995], a link between umbilic geodesics and billiards on $n$-dimensional quadrics and new soliton-like solutions of nonlinear equations in the Dym hierarchy was investigated. The geodesic flows provide the spatial $x$-flow, or the instantaneous profile of the solution of a partial differential equation. When combined with a prescription for advancing the solution in time, a $t$-flow, one is able to determine a class of solutions for the partial differential equation under study (see, for example, Alber and Alber [1985, 1987] and Alber and Marsden [1994]). New classes of solutions can be obtained using deformations of finite dimensional level sets in the phase space. In particular, to obtain soliton, billiard and peakon solutions of nonlinear equations, one applies limiting procedures to the system of differential equations on the Riemann surfaces describing quasiperiodic solutions. To carry this out, one can use the method of asymptotic reduction - for details see Alber and Marsden [1992, 1994] and Alber, Camassa, Holm and Marsden [1994, 1995].

\footnotetext{
${ }^{*}$ Department of Mathematics, University of Notre Dame, Notre Dame, IN 46556. Research partially supported by NSF grants DMS 9403861 and 9508711.

${ }^{\dagger}$ Department of Mathematics, University of Notre Dame, Notre Dame, IN 46556, and the Basic Research Institute in the Mathematical Sciences, Hewlett-Packard Laboratories, Filton Road, Stoke Gifford, Bristol BS12 6QZ, UK. GGL gratefully acknowledges support from BRIMS, Hewlett-Packard Labs and from NSF DMS under grant 9508711.

${ }^{\ddagger}$ Control and Dynamical Systems 104-44, Caltech, Pasadena, CA 91125. Research partially supported by NSF grant DMS 9302992
} 
Elliptic and hyperbolic billiards can be obtained from the problem of geodesics on quadrics by collapsing along the shortest semiaxis - see Alber and Alber [1986] and Moser and Veselov [1991]. This process yields Hamiltonians and first integrals for the resulting billiard problem. A defining characteristic of a billiard solution is that some of the momentum variables experience a jump when a trajectory hits a boundary. In the context of generalized geodesic flows on Riemannian manifolds, these jumps are associated with a reflection map that is used to glue different pieces of the invariant variety in the phase space together to form one symplectic manifold (for generalized geodesic flows and completely integrable systems, see Lazutkin [1993]). These jumps on the invariant variety in the phase space correspond to discontinuities in the instantaneous profile of the solution of the partial differential equation.

In Hamiltonian systems, boundaries of Riemann surfaces are obtained by collapsing several branch points into one or by eliminating two or more branch points. Points obtained in this way are called reflection points or boundaries of Riemann surfaces. Collapsing branch points can change the type of the Riemann surface, for example from a hyperelliptic curve to a logarithmic surface. Boundary conditions must be imposed when reflection points are created. Introducing reflection conditions at boundaries as jumps from one sheet of a Riemann surface to another leads to billiard geodesic flows. The reflection condition then yields billiard-type weak solutions of nonlinear partial differential equations (See Alber, Camassa, Holm, Marsden [1994, 1995]). The interpretation of the jump conditions depends on the particular physical problem and is different in each case. For example, in the case of a three-wave interaction, the jump manifests itself as a shift of the relative phase of the three waves. We describe this example in some detail below to demonstrate a more general approach that can be used to analyze soliton phase functions.

The appearance of monodromy is prevalent in many Hamiltonian systems. A monodromy representation for a complex analytic Hamiltonian system $X_{H}$ is a solution germ that has been analytically continued around a closed loop. Hamiltonian flows are linearized in terms of action-angle representations on Jacobi varieties in many integrable systems. These representations have holomorphic and meromorphic differentials. The presence of these differentials implies the existence of nontrivial fixed points for the action of homotopy classes represented by real periodic orbits (for details see Baider, Churchill and Rod [1990] and Magnus [1976].)

Below we find new classes of solutions of nonlinear partial differential equations with monodromy by constructing associated finite dimensional integrable Hamiltonian systems on Riemannian manifolds. Our approach to evolution equations with monodromy is demonstrated for classes of solutions of the coupled Dym equations. Prior to this, such effects were recognized only in finite dimensional mechanical systems.

\section{Phase Shifts on Riemann Surfaces}

\subsection{Phase Shifts and Monodromy of a Branch Point}

Here we consider an example of a jump in the relative phase of a wave interaction caused by the presence of a branch point on a Riemann surface. The envelope equations for the 
resonant interaction of three waves can be written in the canonical form

$$
\begin{aligned}
\frac{d}{d z} A_{1} & =-i A_{2} A_{3}+i\left(\delta_{1}+\lambda_{1 \beta}\left|A_{\beta}\right|^{2}\right) A_{1}, \\
\frac{d}{d z} A_{2} & =-i A_{1} A_{3}^{*}+i\left(\delta_{2}+\lambda_{2 \beta}\left|A_{\beta}\right|^{2}\right) A_{2}, \\
\frac{d}{d z} A_{3} & =-i A_{1} A_{2}^{*}+i\left(\delta_{3}+\lambda_{3 \beta}\left|A_{\beta}\right|^{2}\right) A_{3},
\end{aligned}
$$

where all quantities are measured in a frame moving with the wave and a repeated index implies summation (see for instance Guckenheimer and Mahalov [1992] or McKinstrie and Luther [1988] and references therein). The $A_{\alpha}$ 's are the action flux amplitudes of each wave, normalized to the initial action flux amplitude of the pump wave. The $\delta_{\alpha}$ 's and the $\lambda_{\alpha \beta}$ 's are the linear and nonlinear phase-mismatch coefficients of each wave. Note that the nonlinear phase-shift coefficients can be chosen to satisfy the symmetry relation $\lambda_{\alpha \beta}=\lambda_{\beta \alpha}$.

This system can be formulated in terms of the Hamiltonian

$$
H=\left(A_{1} A_{2}^{*} A_{3}^{*}+A_{1}^{*} A_{2} A_{3}\right)-\left(\delta_{\alpha}+\frac{1}{2} \lambda_{\alpha \beta}^{\prime}\left|A_{\beta}\right|^{2}\right)\left|A_{\alpha}\right|^{2},
$$

together with Hamilton's equations

$$
i \frac{d}{d z} A_{\alpha}=\frac{\partial H}{\partial A_{\alpha}^{*}} .
$$

In this formulation, the wave amplitudes $A_{\alpha}$ and $A_{\alpha}^{*}$ are canonically-conjugate variables. In addition to the Hamiltonian there are two other constants of the motion,

$$
I=\left|A_{1}\right|^{2}+\left|A_{2}\right|^{2}, \quad J=\left|A_{2}\right|^{2}-\left|A_{3}\right|^{2} .
$$

Writing

$$
A_{\alpha}=F_{\alpha}^{1 / 2} \exp \left(i \phi_{\alpha}\right)
$$

the Hamiltonian takes the form

$$
H=2\left(F_{1} F_{2} F_{3}\right)^{1 / 2} \cos \left(\phi_{1}-\phi_{2}-\phi_{3}\right)-\left(\delta_{\alpha}+\lambda_{\alpha \beta} F_{\beta}\right) F_{\alpha},
$$

where the action flux densities $F_{\alpha}$ and the phases $\phi_{\alpha}$ evolve according to

$$
\frac{d}{d z} F_{\alpha}=-\frac{\partial H}{\partial \phi_{\alpha}}, \quad \frac{d}{d z} \phi_{\alpha}=\frac{\partial H}{\partial F_{\alpha}} .
$$

Using the three constants $H, I$ and $J$, the evolution equation for each action flux density can be rewritten in the potential form

$$
\left(\frac{d}{d z} F_{\alpha}\right)^{2}+Q_{\alpha}\left(F_{\alpha}\right)=0,
$$

where each $Q_{\alpha}$ is a fourth-order polynomial in $F_{\alpha}$. These equations (??) can be transformed into the following problem of inversion:

$$
\int_{z_{0}}^{z} \frac{d F_{\alpha}}{\sqrt{-Q_{\alpha}\left(F_{\alpha}\right)}}=z-z_{0},
$$


which can be solved in terms of Riemann $\theta$-functions on the Jacobi varieties of hyperelliptic curves

$$
W_{\alpha}^{2}+Q_{\alpha}\left(F_{\alpha}\right)=0
$$

Therefore, in this case the exchange of energy and momentum among the three waves is periodic. Once the spatial dependence of $F_{\alpha}$ has been obtained from the appropriate potential equation, $\phi_{\alpha}$, is found by integrating the corresponding phase equation,

$$
\frac{d}{d z} \phi_{\alpha}=P_{\alpha}\left(F_{\alpha}\right)
$$

where each $P_{\alpha}$ is a simple algebraic function of $F_{\alpha}$. For example, setting initial conditions in the form of

$$
F_{1}(0)=1, F_{2}(0)=\epsilon, F_{3}(0)=0,
$$

yields

$$
F_{3}(z)=: F(z), F_{2}(0)=\epsilon+F(z), F_{1}(0)=1-F(z)
$$

and

$$
\left(d_{z} F\right)^{2}=4 F\left((\epsilon+F)(1-F)-F(\delta-\lambda F)^{2}\right)=C(F) .
$$

This also results in the following expression for the relative phase $\Phi=\phi_{1}-\phi_{2}-\phi_{3}$ :

$$
\cos \Phi=-\frac{(\delta-\lambda F) \sqrt{F}}{\sqrt{(1-F)(\epsilon+F)}} .
$$

It follows from (??) that $F$ is changing along a cycle on the Riemann surface

$$
Q^{2}=C(F)
$$

over the cut between two roots of the polynomial $C(F)$. In the process of investigating the dynamics of the phase function $\Phi$ one also has to deal with the multivaluedness of the expression on the righthand side of (??). The value of $F$ is defined on the Riemann surface (??) and therefore $\cos \Phi$ should be considered on a covering of this Riemann surface. This yields relative phase shifts. For example, after going through the branch point at $F=0$, $\cos \Phi$ goes from one sheet of the covering to another identified with $(\sqrt{F})$ and $(-\sqrt{F})$ respectively. This yields a shift of $\pi$ in $\Phi$.

When $\delta=\lambda$ the maximum energy transfer among the waves can be achieved. The point $F=1$ becomes a branch point, and the expression for the relative phase becomes

$$
\cos \Phi=-\frac{\delta \sqrt{F(1-F)}}{\sqrt{(\epsilon+F)}} .
$$

Remark 1. The existence of the general phenomenon of a geometric phase (as in Berry and Hannay [1988] and Marsden, Montgomery and Ratiu [1990]) can be demonstrated as follows (see Alber, Luther and Marsden [1996]). We first notice that $\theta=z-z_{0}$ from (??) is an angle variable for our problem. For a periodic orbit we can write

$$
\Delta_{g} \theta=\oint_{\gamma} d X\left\langle\partial_{X} \theta,\right\rangle
$$


and $\Delta_{g} \theta$ is a nontrivial geometric phase, where $X$ denotes the parameters of the system, namely the coefficients of the polynomial $C(F), \gamma$ is a closed cycle in the space of parameters and where $\langle$,$\rangle denotes averaging along the periodic orbit, which is parametrized by the$ action. This action is the constant term in the polynomial $C(F)$. This will also result in a geometric phase for the $\phi$.

Remark 2. Variations in the medium that hosts the wave result in changes in the parameters $\delta$ and $\lambda$. Since these parameters along with $\epsilon$ determine the location of the fixed points and therefore the homoclinic point, their motion during the dynamics can significantly alter the dynamics of the waves. It is possible to vary $\delta$ and $\lambda$ so that the phase point of a wave makes a circuit around one of the fixed points resulting in the shift associated with the monodromy.

\subsection{Phase Shifts and Billiards}

Now we demonstrate that in the limiting case $\epsilon \rightarrow 0$ the phase jump is generated by reflection on the boundary of a billiard system on a Riemann surface. When $\epsilon \rightarrow 0$ (infinite-period limit), the Homoclinic orbit is recovered. This is associated with a singular leaf of the Jacobian foliation in the phase space. A homoclinic orbit is obtained when the daughter waves initially have exponentially small amplitudes for $\xi \rightarrow-\infty$. In this limit the three waves exchange their energy exactly once and the recurrence time is infinity. Note also that the position of the homoclinic is altered by changing the parameters $\delta$ and $\lambda$.

The potential for wave three reduces in the limit $\epsilon \rightarrow 0$ to

$$
Q^{2}=4 F^{2}\left((1-F)-(\delta-\lambda F)^{2}\right)
$$

and equations for $\Phi$ and $F$ become

$$
\begin{aligned}
\xi+\xi_{0} & =\int_{\xi^{0}}^{\xi} \frac{d F}{2 F \sqrt{(1-F)-(\delta-\lambda F)^{2}}} \\
\cos \Phi & =\frac{-(\delta-\lambda F)}{(1-F)^{1 / 2}} .
\end{aligned}
$$

The Riemann surface for $F$ has a logarithmic singularity at $F=0$. At the same time the branch point at $F=0$ in the expression for $\cos \Phi$ cancels out. Nevertheless, the sign of the expression changes every time $F$ reaches $F=0$ due to the reflection condition on the boundary $F=0$. This yields a shift in the relative phase $\Phi$.

\section{Monodromy in Solutions of Nonlinear PDE's}

In this section we proceed with our discussion by describing special solutions of nonlinear equations with monodromy. Examples include parametrized classes of solutions of completely integrable equations such as the coupled Dym and the NLS equations. The connection to Hamiltonian systems on Riemann surfaces in these examples is more complicated than in the case of the three-wave equations, but it can be established, for example, using the method of generating equations. 
We begin by considering the spectral problem for an associated Schrödinger operator,

$$
L=-\frac{\partial^{2}}{\partial x^{2}}+V(x, t, E)
$$

where $E$ is a parameter and

$$
V(x, t, E)=\sum_{j=-l}^{m} V_{j}(x, t) E^{j} .
$$

In some cases, such as the KdV equation, $E$ appears as an eigenvalue and one ultimately equates the potential with a solution of the nonlinear equation itself. In the case of the nonlinear Schrödinger equation, the solution, $Q$, and the potential, $V$, are related in a slightly more complicated way. To carry out the procedure, one looks for a solution $A$ of the Lax system

$$
\left.\begin{array}{rl}
L \psi & =0 \\
\left(\frac{\partial L}{\partial t}+[L, A]\right) \psi & =0
\end{array}\right\}
$$

of the form

$$
A=B_{n} \frac{\partial}{\partial x}-\frac{1}{2} \frac{\partial B_{n}}{\partial x} .
$$

Substituting the given form of $A$ into the Lax system, one gets

$$
\frac{\partial V}{\partial t}=-\frac{B_{n}^{\prime \prime \prime}}{2}+2 B_{n}^{\prime} V+B_{n} V^{\prime}
$$

where the prime denotes $\partial / \partial x$ and $B_{n}=\prod_{j=1}^{n}\left(E-\mu_{j}(x, t)\right)$. Equation (??) is called the generating equation. Suppose $\partial V / \partial t=0$, then the equation can be integrated resulting in the following stationary equation:

$$
-B_{n}^{\prime \prime} B_{n}+\frac{B_{n}^{\prime 2}}{2}+2 B_{n}^{2} V=K(E)
$$

where $K(E)$ is a rational function of $E$ with constant coefficients. In the inverse scattering transform method it is called the spectral polynomial (see Moser [1981]).

To describe the dynamics of $B_{n}$ we use the following procedure. We suppose that at each instant of time, $B_{n}$ is a solution of the stationary generating equation. The evolution of $B_{n}$ in time is then determined by

$$
\dot{B}_{n}=B_{n}^{\prime} B_{l}-B_{l}^{\prime} B_{n}, \quad \text { i.e., } \quad \frac{\partial}{\partial t}\left(\frac{1}{B_{n}}\right)=\frac{\partial}{\partial x}\left(\frac{B_{l}}{B_{n}}\right),
$$

where $B_{l}$ is a solution of the dynamical generating equation (??). For particular choices of the form of the potential $V$, equations (??) and (??) generate a hierarchy of integrable equations.

Using (??) and (??), finite dimensional Hamiltonian systems in the $\mu$-representation for solutions of the nonlinear equations in the hierarchy are obtained after substituting $E=\mu_{j}$, where $j=1, \ldots n$. The $x$-flow is given by the system of equations $\mu_{j}^{\prime}$ and the 
$t$-flow is given by the system $\dot{\mu}_{j}$. The $\mu$-representations have complete sets of first integrals in the form of Riemann surfaces. The Abel map is frequently used for these Riemann surfaces, and it linearizes the Hamiltonian flows on the associated Jacobi varieties. Recall from Ercolani [1989] and Alber et al. [1994, 1995] that the Abel map can also be viewed as a set of the complex angle variables called the complex angle representation of the problem. Continuous variation of the first integrals results in deformations of the level sets. Some of these deformations lead to singular cases for which one must introduce new types of angle representations on singular level sets. Examples of this situation are provided by homoclinic and soliton Hamiltonian flows; these systems usually have phase shifts produced by monodromy effects.

\subsection{Phase Shifts for the NLS Equation}

In this section we describe phase shifts produced by the interaction of fundamental solutions of the NLS equation. To do so we use the angle representation obtained in Alber and Marsden [1992, 1994].

Applying the general formalism of generating equations and asymptotic reduction (from Alber and Marsden [1992]) gives the angle representation that describes the collision of two standard solitons of the (f)NLS equation, namely

$$
\begin{gathered}
\theta_{1}=-\frac{1}{4 y_{1}} \int_{\mu_{j}^{0}}^{\mu_{1}}\left(\frac{1}{\left(\mu_{1}-a_{1}\right)}-\frac{1}{\left(\mu_{1}-\bar{a}_{1}\right)}\right) d \mu_{1}+\phi_{1}\left(\mu_{2}, \mu_{3}\right)=x+v_{1} t, v_{1}=2 x_{1} \\
\theta_{2}=-\frac{1}{4 y_{2}} \int_{\mu_{2}^{0}}^{\mu_{2}}\left(\frac{1}{\left(\mu_{2}-a_{2}\right)}-\frac{1}{\left(\mu_{2}-\bar{a}_{2}\right)}\right) d \mu_{2}+\phi_{2}\left(\mu_{2}, \mu_{3}\right)=x+v_{2} t, v_{2}=2 x_{2}, \\
\theta_{3}=-\frac{1}{2\left(y_{1}-y_{2}\right)} \int_{\mu_{3}^{0}}^{\mu_{3}}\left(\frac{1}{\left(\mu_{3}-a_{1}\right)}-\frac{1}{\left(\mu_{3}-a_{2}\right)}\right) d \mu_{3}+\phi_{3}\left(\mu_{2}, \mu_{3}\right)=x+v_{3} t+i \operatorname{Im} \theta_{3} .
\end{gathered}
$$

Where

$$
v_{3}=\frac{2\left(y_{1} x_{1}-y_{2} x_{2}\right)}{\left(y_{1}-y_{2}\right)}
$$

and $a_{1}=x_{1}+i y_{1}$ and $a_{2}=x_{2}+i y_{2}$ are elements of the discrete spectrum of the differential operator $L$. We assume that $x_{2}<x_{1}$ and $y_{2}<y_{1}$, which implies that $v_{2}<v_{1}$.

The complex phase functions associated with the angle variables are defined by

$$
\begin{aligned}
\phi_{1}(x, t)= & -\frac{1}{4 y_{1}}\left(\int_{\mu_{2}^{0}}^{\mu_{2}}\left(\frac{1}{\left(\mu_{2}-a_{1}\right)}-\frac{1}{\left(\mu_{2}-\bar{a}_{1}\right)}\right) d \mu_{2}\right), \\
& +\frac{1}{4 y_{1}}\left(\int_{\mu_{3}^{0}}^{\mu_{3}}\left(\frac{1}{\left(\mu_{3}-a_{1}\right)}-\frac{1}{\left(\mu_{3}-\bar{a}_{1}\right)}\right) d \mu_{3}\right)
\end{aligned}
$$

and

$$
\begin{aligned}
\phi_{2}(x, t)= & -\frac{1}{4 y_{2}}\left(\int_{\mu_{1}^{0}}^{\mu_{1}}\left(\frac{1}{\left(\mu_{1}-a_{2}\right)}-\frac{1}{\left(\mu_{1}-\bar{a}_{2}\right)}\right) d \mu_{1}\right) \\
& +\frac{1}{4 y_{2}}\left(\int_{\mu_{3}^{0}}^{\mu_{3}}\left(\frac{1}{\left(\mu_{3}-a_{2}\right)}-\frac{1}{\left(\mu_{3}-\bar{a}_{2}\right)}\right) d \mu_{3}\right) .
\end{aligned}
$$


The phase shifts of solitons are related to the monodromy of $\phi_{1}$ and $\phi_{2}$. The phase shifts are obtained by considering the asymptotic behavior of the relative phase function, $\phi(x, t)=$ $\phi_{1}(x, t)-\phi_{2}(x, t)$. Notice that each of the $\mu$ variables is defined on a logarithmic Riemann surface and $\mu_{1}, \mu_{2}$ and $\mu_{3}$ describe a dynamical system with two homoclinic points. Phase shifts, or jumps in the values of phase functions, accrue precisely when the system goes through one of these points. This situation is, therefore, very similar to the three-wave interaction.

\subsection{Special Solutions for the Coupled Dym System}

In what follows, we introduce a Hamiltonian system for the set of quasiperiodic solutions of the so-called coupled Dym system. When applied to this Hamiltonian system, different limiting procedures (involving the coalescence of roots of the basic polynomial of the spectral curve) yield special solutions with monodromy, billiard solutions and umbilic geodesic flows (in the presence of potentials) on associated limiting Riemann surfaces.

The time evolution of eigen functions of the generalized Schrödinger equations and the multi-Hamiltonian structures associated with the coupled Dym system were investigated in Antonowicz and Fordy [1988, 1989] using an algebraic approach. Recall from these papers that the potential associated with the coupled Dym system

$$
\left.\begin{array}{l}
\frac{\partial u}{\partial t}=\frac{1}{4} u^{\prime \prime \prime}-\frac{3}{2} u u^{\prime}+v^{\prime}, \\
\frac{\partial v}{\partial t}=-u^{\prime} v-\frac{1}{2} u v^{\prime},
\end{array}\right\}
$$

has the form $V=u(x)+\lambda+v(x) / \lambda$ and has a pole in the spectral parameter. Using the method of Alber, Camassa, Holm and Marsden [1994, 1995] one obtains the stationary $\mu$-flow,

$$
\mu_{j}^{\prime}=\frac{1}{\prod_{r \neq j}^{n}\left(\mu_{j}-\mu_{r}\right)} \sqrt{\frac{C\left(\mu_{j}\right)}{\mu_{j}}}, j=1, \ldots, n,
$$

for this integrable problem after substituting $V$ into the generating equation (??). Each of the $\mu$ variables is defined on a copy of the Riemann surface

$$
\Re: P^{2}=\frac{C(\mu)}{\mu}=-\frac{L_{0}^{2}}{\mu} \prod_{k=1}^{2 n+2}\left(\mu-m_{k}\right) .
$$

Recall that the $\mu$ variables move along cycles on the corresponding Riemann surface (??) over the prohibited zones (that is, over the basic cuts between $m_{2 j}$ and $m_{2 j-1}$ on the Riemann surface). The system (??) is a Hamiltonian system with Hamiltonian

$$
H=\sum_{j=1}^{n} \frac{\left(P_{j}^{2}-\frac{C\left(\mu_{j}\right)}{\mu_{j}}\right)}{\prod_{r \neq j}^{n}\left(\mu_{j}-\mu_{r}\right)}, \quad j=1, \ldots, n,
$$


and the set of first integrals

$$
P_{j}^{2}=\frac{C\left(\mu_{j}\right)}{\mu_{j}}, j=1, \ldots, n
$$

Here the system is degenerate because the genus of the Riemann surface (??) is $(n+1)$, yet we have only $n \mu$ variables. This difference produces a degeneracy in the problem of inversion. It can be resolved by introducing an additional $\mu$ variable, $\mu_{n+1}$, and solving the problem of inversion in terms of Riemann $\theta$-functions on the $(n+1)$-dimensional Jacobian of the hyperelliptic curve

$$
\Re: P^{2}=C(\mu) \mu,
$$

which is a torus of genus $g=n+1$. In this way the following problem of inversion is obtained in the complex angle representation,

$$
\alpha_{k}=\sum_{j=1}^{n+1} \int_{\mu_{j}^{0}}^{\mu_{j}} \frac{\mu_{j}^{k+1} d \mu_{j}}{\sqrt{C\left(\mu_{j}\right) \mu_{j}}}=\delta_{k}^{n-1} x+\delta_{k}^{n-2} t+\alpha_{k}^{0}, \quad k=0, \ldots, n
$$

where $\alpha_{k}^{0}$ are constants, $\delta_{k}^{n-1}, \delta_{k}^{n-2}$ are Kronecker delta's and each $\mu_{j}$ is defined on a copy of the Riemann surface (??). The above integrals are taken along cycles $a_{j}$ over basic cuts on the Riemann surface. The solution of the Hamiltonian system is obtained by setting $\mu_{n+1}=0$.

After applying a limiting procedure similar to that described in Alber, Camassa, Holm and Marsden [1995] for the Dym equation and fixing one of the $\mu$ variables, the system (??) leads to the so-called umbilic angle representation. Alber, Camassa, Holm and Marsden [1995] shows that these representations generate a class of umbilic solitons and billiard solutions of the coupled system of partial differential equations that were described in the introduction.

On the other hand, setting $m_{2 n+2}=m_{2 n+1}=d$ in the initial Hamiltonian system (without the additional $\mu$ variable) yields a well-defined system of inversion with monodromy in the space of parameters. Namely, moving $d$ along a certain closed loop in the space of parameters can lead to a nontrivial shift in action angle variables. This phenomenon is caused by a singularity on the associated Riemann surface and can be demonstrated as follows. In the 1-dimensional case, the limiting process $m_{3}, m_{4} \rightarrow d$ yields the angle representation

$$
\alpha_{1}=\int_{\mu_{1}^{0}}^{\mu_{1}} \frac{\mu_{1} d \mu_{1}}{\left(\mu_{j}-d\right) \sqrt{-\mu_{1}\left(\mu_{1}-m_{1}\right)\left(\mu_{1}-m_{2}\right)}}=L_{0} x+\alpha_{1}^{0} .
$$

In the case of a genus 3 initial Riemann surface, the limiting angle representation is as follows

$$
\begin{aligned}
& \alpha_{1}=-\frac{\partial S}{\partial \beta_{1}}=\int_{\mu_{1}^{0}}^{\mu_{1}} \frac{\mu_{1} d \mu_{1}}{\left(\mu_{1}-d\right) \sqrt{C_{5}\left(\mu_{1}\right)}}+\int_{\mu_{2}^{0}}^{\mu_{2}} \frac{\mu_{2} d \mu_{2}}{\left(\mu_{2}-d\right) \sqrt{C_{5}\left(\mu_{2}\right)}}=\alpha_{1}^{0} . \\
& \alpha_{2}=-\frac{\partial S}{\partial \beta_{2}}=\int_{\mu_{1}^{0}}^{\mu_{1}} \frac{\mu_{1} d \mu_{1}}{\sqrt{C_{5}\left(\mu_{1}\right)}}+\int_{\mu_{2}^{0}}^{\mu_{2}} \frac{\mu_{2} d \mu_{2}}{\sqrt{C_{5}\left(\mu_{2}\right)}}=L_{0} x+\alpha_{2}^{0}
\end{aligned}
$$


where

$$
S=\int_{\mu_{1}^{0}}^{\mu_{1}} \frac{\sqrt{C_{5}\left(\mu_{1}\right)} d \mu_{1}}{\left(\mu_{1}-d\right)}+\int_{\mu_{2}^{0}}^{\mu_{2}} \frac{\sqrt{C_{5}\left(\mu_{2}\right)} d \mu_{2}}{\left(\mu_{2}-d\right)}
$$

is an action function (the generating function of a canonical transformation) and

$$
C_{5}(\mu)=-\mu\left(\beta_{2}(\mu-b)+\beta_{1}+R_{4}(\mu)\right)=-\mu\left(\mu-m_{1}\right)\left(\mu-m_{2}\right)\left(\mu-m_{3}\right)\left(\mu-m_{4}\right) .
$$

The variables $\mu_{1}$ and $\mu_{2}$ move along cycles $a_{1}$ and $a_{2}$ over the cuts $\left[m_{1}, m_{2}\right]$ and $\left[m_{3}, m_{4}\right]$ on the Riemann surface $W^{2}=C_{5}(\mu)$. There is also a singularity at $\mu=d$. Transport of a system of canonical action-angle variables, which linearize the Hamiltonian flow, along a certain loop in the space of parameters $\left(d, m_{j}\right)$ in a way similar to the case of the spherical pendulum and some other integrable systems with monodromy (see, for example, Duistermaat [1980] and Bates and Zou [1993]) will result in a nontrivial shift, which is a manifestation of the monodromy phenomenon. This can be demonstrated as follows. Canonical actions are calculated in terms of periods of the holomorphic differential

$$
I_{j}=\oint_{a_{j}} d S=\oint_{a_{j}} \frac{\sqrt{C_{5}(\mu)} d \mu}{(\mu-d)}
$$

along cycles $a_{j}$ on the Riemann surface (a torus); for details see Arnold [1978]. Now suppose initially that $d$ does not belong to either of the cycles $a_{1}$ or $a_{2}$. By moving $d$ along a closed loop on the Riemann surface such that it encircles one of the branch points, $m_{j}$, a shift in the action variable is produced that is given by the residue of the integrand at $\mu=d$.

Lastly, the method of Alber et al. [1994, 1995] of associating solutions of nonlinear partial differential equations with finite dimensional Hamiltonian systems on Riemann surfaces leads to the construction of a class of solutions of nonlinear partial differential equations with monodromy.

\section{Coupled Nonlinear Schrödinger Equations}

Our last example is provided by the coupled NLS equations. A pair of distinct wave packets propagating under the action of dispersion and cubic nonlinearity are modeled by the coupled nonlinear Schrödinger equation (CNLS),

$$
\begin{aligned}
& {\left[i\left(\partial_{t}+v_{1} \partial_{z}\right)+\lambda_{11}\left|A_{1}\right|^{2}+\lambda_{12}\left|A_{2}\right|^{2}\right] A_{1}+\Delta_{1} A_{2}=0} \\
& {\left[i\left(\partial_{t}+v_{2} \partial_{z}\right)+\lambda_{21}\left|A_{1}\right|^{2}+\lambda_{22}\left|A_{2}\right|^{2}\right] A_{2}+\Delta_{2} A_{1}=0 .}
\end{aligned}
$$

The CNLS equations have several different forms and arise in plasma physics, fluid dynamics and nonlinear optics. The CNLS models the coupling of wave envelopes propagating in two coupled nonlinear waveguides (dual-core fibers), in two separate polarization modes in a single waveguide, or at two distinct frequencies in the same waveguide and polarization mode. Understanding the integrable Manakov system [1974], which is a special case of the system (??) and (??) as well as its near-integrable counterparts is of basic interest for the analysis of problems in optical communications and switching. 
Stationary solutions of the CNLS are found by taking

$$
(A, B)=\left(q_{1}(t), q_{2}(t)\right) \exp (i \Omega z)
$$

That is, $\left(q_{1}(t), q_{2}(t)\right)$ are the complex amplitude profiles of envelopes in two modes that couple nonlinearly as described above. Solutions $\left(q_{1}(t), q_{2}(t)\right)$ can be found using algebraic geometric methods by choosing

$$
B(E, x)=1-\frac{q_{1}^{2}}{2\left(E-l_{1}\right)}-\frac{q_{2}^{2}}{2\left(E-l_{2}\right)}=\frac{\left(E-\mu_{1}\right)\left(E-\mu_{2}\right)}{2\left(E-l_{1}\right)\left(E-l_{2}\right)} \quad \text { and } \quad V=u(x)+E,
$$

in (??). This yields a system of two coupled ODE's for $q_{1}$ and $q_{2}$. Namely, substituting (??) into (??), multiplying by $\left(E-l_{1}\right)^{2}\left(E-l_{2}\right)^{2}$ and setting separately $E \rightarrow l_{1}$ and $E \rightarrow l_{2}$ yields

$$
\left.\begin{array}{l}
q_{1}^{\prime \prime}+\left(q_{1}^{2}+q_{2}^{2}\right) q_{1}=l_{1} q_{1} \\
q_{2}^{\prime \prime}+\left(q_{1}^{2}+q_{2}^{2}\right) q_{2}=l_{2} q_{2}
\end{array}\right\}
$$

In Moser [1981] it was shown that a similar flow provides solutions for the C. Neumann problem on a 2-sphere: $q_{1}^{2}+q_{2}^{2}+q_{3}^{2}=1$, in 3-dimensional space $\mathbf{R}^{3}$ and can be linked with the geodesic flow on an ellipsoid. In the present context, we deal with two independent variables $q_{1}$ and $q_{2}$ without any constraints. This system can be associated with geodesics in $\mathbf{R}^{2}$.

Solutions of (??) can be obtained using $\mu$ representations on Riemann surfaces. Setting $E=\mu_{1}$ or $E=\mu_{2}$ in (??) yields

$$
\left.\begin{array}{l}
\mu_{1}^{\prime}=\frac{\sqrt{-\left(\mu_{1}-l_{1}\right)\left(\mu_{1}-l_{2}\right)\left(\mu_{1}-l_{3}\right)\left(\mu_{1}-l_{4}\right)\left(\mu_{1}-l_{5}\right)}}{\mu_{1}-\mu_{2}} \\
\mu_{2}^{\prime}=\frac{\sqrt{-\left(\mu_{2}-l_{1}\right)\left(\mu_{2}-l_{2}\right)\left(\mu_{2}-l_{3}\right)\left(\mu_{2}-l_{4}\right)\left(\mu_{2}-l_{5}\right)}}{\mu_{2}-\mu_{1}}
\end{array}\right\}
$$

This is a hamiltonian system on a Riemann surface of genus two, and it describes the stationary quasiperiodic KdV flow. In Christiansen et al. [1995] elliptic solutions of the Jacobi problem of inversion associated with (??) is linked with the Treibich-Verdier potentials [1991] for the Schrödinger equation.

The following limiting process $l_{1} \rightarrow 0, l_{2}, l_{3} \rightarrow a_{1}$ and $l_{4}, l_{5} \rightarrow a_{2}$ when applied to (??), yields homoclinic orbits

$$
\left.\begin{array}{r}
\mu_{1}^{\prime}=\frac{\sqrt{-\mu_{1}}\left(\mu_{1}-a_{1}\right)\left(\mu_{1}-a_{2}\right)}{\mu_{1}-\mu_{2}} \\
\mu_{2}^{\prime}=\frac{\sqrt{-\mu_{2}}\left(\mu_{2}-a_{1}\right)\left(\mu_{2}-a_{2}\right)}{\mu_{2}-\mu_{1}}
\end{array}\right\}
$$


Lastly, solutions of the system (??) are related to $\mu$ variables on Riemann surfaces as follows:

$$
\left.\begin{array}{l}
q_{1}=\sqrt{\frac{-\left(l_{1}-\mu_{1}\right)\left(l_{1}-\mu_{2}\right)}{l_{1}-l_{2}}} \\
q_{2}=\sqrt{\frac{-\left(l_{2}-\mu_{1}\right)\left(l_{2}-\mu_{2}\right)}{l_{2}-l_{1}} .}
\end{array}\right\}
$$

The finite dimensional invariant variety for $q_{1}$ and $q_{2}$ is a covering of the Riemann surface which is associated with the representation of the system in terms of $\mu$ variables. The structure of this variety can be investigated using methods of algebraic geometry (see Christiansen et al. [1995]); monodromy of the branch points plays a central role in this approach.

\section{References}

M.J. Ablowitz and H. Segur [1981], Solitons and the Inverse Scattering Transform, SIAM, Philadelphia.

M.S. Alber and S.J. Alber [1986], On Hamiltonian formalism for finite-zone solutions of nonlinear integrable equations, Proc.VIII Int.Cong.on Math. Physics, Marseille, France (World Scientific) $447-462$.

M.S. Alber and S.J. Alber [1985], Hamiltonian formalism for finite-zone solutions of integrable equations, C.R. Acad. Sc. Paris 301, $777-781$.

M. S. Alber and S. J. Alber [1987], Hamiltonian formalism for nonlinear Schrödinger equations and sine-Gordon equations, J. London Math. Soc. 36, 176 - 192.

M.S. Alber, R. Camassa, D.D. Holm and J.E. Marsden [1994], The geometry of peaked solitons and billiard solutions of a class of integrable partial differential equation's, Lett. Math. Phys. 32, $137-151$.

M.S. Alber, R. Camassa, D.D. Holm and J.E. Marsden [1995], On the link between umbilic geodesics and soliton solutions of nonlinear partial differential equation's, Proc. Roy. Soc. Lond. A 450677 - 692 .

M.S. Alber, G.G. Luther and J.E. Marsden [1996], Geometric phase in wave mixing and soliton interactions, BRIMS, Hewlett Packard Laboratories preprint.

M.S. Alber and J.E. Marsden [1992], On geometric phases for soliton equations, Commun. Math. Phys., 149, $217-240$.

M.S. Alber and J.E. Marsden [1994a], Geometric phases and monodromy at singularities, N.M. Ercolani et al., eds., NATO ASI Series B (Plenum Press, New York) 320273 296. 
M.S. Alber and J.E. Marsden [1994b], Resonant Geometric Phases for Soliton Equations, Fields Institute Commun. $31-26$.

M. Antonowicz and A.P. Fordy [1988], Coupled Harry Dym equations with multi-Hamiltonian structures, J. Phys. A 21 L269-L275.

M. Antonowicz and A.P. Fordy [1989], Factorisation of energy dependent Schrödinger operators: Miura maps and modified systems, Commun. Math. Phys. $124465-486$.

V.I. Arnold [1989], Mathematical Methods of Classical Mechanics, (Springer-Verlag: New York, Heidelberg, Berlin).

A. Baider, R. C. Churchill and D. L. Rod [1990], Monodromy and nonintegrability in complex Hamiltonian systems, J Dyn. Diff. Equations 2, $451-481$.

L. Bates and M. Zou [1993], Degeneration of Hamiltonian monodromy cycles, Nonlinearity 6, $313-335$.

Berry, M. and J. Hannay [1988], Classical non-adiabatic angles, J. Phys. A. Math. Gen. $\mathbf{2 1}, 325-333$.

H. Braden [1982], A completely integrable mechanical system, Lett. Math. Phys. $6449-$ 452.

P.L. Christiansen, J.C. Eilbeck, V.Z. Enolskii and N.A. Kostov [1995], Quasiperiodic solutions of the coupled nonlinear Schrödinger equations, preprint.

J.J. Duistermaat [1980], On global action-angle coordinates, Comm. Pure Appl. Math. 23687 - 706.

N. Ercolani [1989], Generalized theta functions and Homoclinic varieties, Proc. Symp. Pure Appl. Math. 4987.

J. Guckenheimer and A. Mahalov [1992], Resonant triad interactions in symmetric systems, Physica D 54, 267 - 310.

V. F. Lazutkin [1993], KAM Theory and Semiclassical Approximations to Eigenfunctions, A Series of Modern Surveys in Mathematics, Springer-Verlag Berlin Heidelberg.

W. Magnus [1976], Monodromy groups and Hill's equations, Commun. Pure Appl.Math. 29, $701-716$.

S.V. Manakov [1974], On the theory of two-dimensional stationary self-focusing of electromagnetic waves, Sov. Phys. JETP 38, $248-253$.

J.E. Marsden, R. Montgomery and T.S. Ratiu [1990], Reduction, symmetry and phases in mechanics. Memoirs AMS 436.

C.J. McKinstrie [1988], Relativistic solitary-wave solutions of the beat-Wave equations, Phys. Fluids 31, $288-297$. 
C.J. McKinstrie and G.G. Luther [1988], Solitary-wave solutions of the generalized threewave and four-wave equations, Phys. Lett. A 127, $14-18$.

C.J. McKinstrie and X.D. Cao [1993], The nonlinear detuning of three-wave interactions, J. Opt. Soc. Am. B 10, $898-912$.

J. Moser [1981], Integrable Hamiltonian systems and spectral theory, Lezioni Fermiane, Accademia Nazionale dei Lincei, Pisa.

J. Moser and A.P. Veselov [1991], Discrete versions of some classical integrable systems and factorization of matrix polynomials, Commun. Math. Phys., 139, $217-243$.

A.C. Newell [1985], Solitons in Mathematics and Physics, Regional Conf. Series in Appl. Math. 48, SIAM, Philadelphia.

A. Treibich and J-L Verdier [1991], Solitons elliptiques, in Special volume for 60th Anniversary of Professor A. Grothendieck, Birkhaüser, Boston. 